\title{
Association between Obesity and Carotid Intima-Media Thickness in Korean Office Workers: The Mediating Effect of Physical Activity
}

\author{
Youngyun Jin, Donghyun Kim, Jinkyung Cho $\mathbb{D}$, Inhwan Lee $\mathbb{D}$, \\ Kyuhwan Choi, and Hyunsik Kang $\mathbb{D}$
}

College of Sport Science, Sungkyunkwan University, Suwon, Republic of Korea

Correspondence should be addressed to Hyunsik Kang; hkang@skku.edu

Received 27 February 2018; Revised 2 July 2018; Accepted 16 July 2018; Published 1 August 2018

Academic Editor: Rei Shibata

Copyright (C) 2018 Youngyun Jin et al. This is an open access article distributed under the Creative Commons Attribution License, which permits unrestricted use, distribution, and reproduction in any medium, provided the original work is properly cited.

\begin{abstract}
Background. Obesity and physical inactivity are associated with higher prevalence of cardiovascular disease (CVD). Objective. This study investigated the association between obesity and carotid intima-media thickness (CIMT) stratified by physical activity (PA) in Korean office workers. Methods. Data obtained from 914 office workers aged 21-60 years (347 women) were used. Resting blood pressures, body mass index (BMI), and waist-to-height ratio (WHtR) were measured. PA was assessed using the international physical activity questionnaire. CIMT was assessed with a carotid artery ultrasonography. Logistic regression analysis was used to estimate the odds ratio (OR) and 95\% confidence interval (CI) of obesity stratified by weekly PA for an abnormally increased CIMT. Results. Logistic regression analyses showed that those who were overall obese $(\mathrm{OR}=2.50,95 \% \mathrm{CI}=1.60-3.91, \mathrm{P}<0.001)$ or central obese $(\mathrm{OR}=2.08,95 \% \mathrm{CI}=1.29-3.40, \mathrm{P}=0.003)$ had significantly higher estimated risks of having an abnormally increased CIMT even after adjustments for age, sex, smoking, alcohol consumption, resting blood pressures, and history of hypertension, diabetes, and hyperlipidemia, as compared with those who were not overall or central obese $(\mathrm{OR}=1)$. A multivariate linear regression suggested that age $(\mathrm{P}<0.001)$, sex $(\mathrm{P}=0.002)$, hypertension $(\mathrm{P}=0.014)$, smoking $(\mathrm{P}=0.054)$, BMI $(\mathrm{P}<0.001)$, and physical activity $(\mathrm{P}=0.011)$ were important determinants of abnormally elevated CIMT in this study population. Conclusion. The current findings suggest that the risk of obesity for an abnormally increased CIMT is significantly modulated by demographics as well as lifestyle-related risk factors including smoking and physical inactivity in Korean office workers.
\end{abstract}

\section{Introduction}

The Korean lifestyle is characterized as largely sedentary, with many people failing to engage in sufficient moderate or vigorous physical activity (PA) [1]. In particular, office workers in Korea spend most of their work hours in sedentary behaviors in conjunction with an unhealthy lifestyle featuring of heavy drinking and smoking and job-related stresses $[2,3]$. Consequently, they are vulnerable to the development of metabolic and cardiovascular disorders, implying an urgent need for more attention and improvement.

Cardiovascular disease (CVD) is a leading cause of death in the general population. Carotid intima-media thickness (CIMT) holds prognostic information for future CVD [4] and is associated with the extent of atherosclerotic lesions
[5]. An abnormally increased CIMT correlates with higher prevalence and incidence of CVD [6,7]. On the other hand, a cholesterol-lowering therapy such as administration of statins may stabilize the carotid atherosclerosis [8] and/or regresses its progression [9].

Obesity is a major modifiable risk factor for CVD [10]. Body mass index (BMI) indicative of overall obesity is positively associated with an abnormally increased CIMT [11]. In addition, waist-to-height ratio (WHtR) indicative of body fat distribution is also closely associated with an abnormally increased CIMT as well as impaired arterial distensibility and compliance [12]. Along with obesity, low PA and sedentary behaviors are associated with higher CVD risk [13]. Thus, the world health organization (WHO) recognizes physical inactivity as the fourth leading risk factor for death globally, 
with an estimated 3.2 million deaths attributed to insufficient PA every year [10]. On the other hand, it is well known that regular PA has the potential to benefit cardiovascular health including atherosclerosis [14] as well as cardiovascular function, body composition, and metabolic conditions [15]

In Korea, office workers are known to be among the unhealthiest group of people due to their sedentarism characterized by prolonged sitting and lack of physical activity in conjunction with overweight and obesity [16]. However, there is a lack of research on office workers with respect to metabolic and cardiovascular health. Therefore, this study aimed to investigate the relationship between overall and central obesity and increased CIMT stratified by weekly PA levels in a sample of Korean office workers.

\section{Methods}

2.1. Subjects. In a cross-sectional design, a total of 982 adults (aged 21-60 years) voluntarily participated in this study, which was conducted as part of the annual health screening at local health centers between March 2015 and October 2016. The exclusion criteria included age of either younger than 21 years or older than 60 years, diagnosed CVD, and history of CVD or stroke. CVD was defined as present if the participant had a history of coronary artery disease, myocardial infraction, atrial fibrillation and other arrhythmias, and heart valve disease.

Data was collected during two separate visits. At the first visit, 589 men ( 8 missing) and 376 women (9 missing) completed demographic assessments, and medical history of hypertension, diabetes mellitus, and hyperlipidemia were obtained from a standardized self-administered questionnaire. The second visit involved an overnight fast after which laboratory tests were performed to evaluate anthropometrics, including height, weight, and waist circumference (WC), cardiovascular risk factors, and CIMT. During the second visit, 22 men and 29 women did not show up or refused to complete assessment of anthropometrics and risk factors or CIMT measurement. Consequently, 914 (567 men/347 women) completed all the measurements and were included in the final data analysis. Informed consent was obtained from all subjects prior to study participation. The Sungkyunkwan University Institutional Review Board, in accordance with the World Medical Association Declaration of Helsinki, approved the study protocol (SKKU 2015-09-001-005).

2.2. Assessment of Lifestyle Risk Factors. Lifestyle factors measured in this study included alcohol consumption (frequency per week) and smoking status. Smoking was defined as smoking more than 1 cigarette per day for at least 1 year, and participants were categorized as never smokers, ever smokers (ceased smoking for at least 6 months), and current smokers. Resting blood pressure (BP) was measured with an automatic BP instrument (Jawon Medical Co., Kyungsan, Republic of Korea) with subjects in seated position, with the arm at heart level and resting on the armrest of a chair.
2.3. Measurement of PA, Anthropometrics, and Obesity. The development of the International Physical Activity Questionnaire (IPAQ) commenced in Geneva in 1998 and was followed by extensive reliability and validity testing undertaken across countries [17]. The IPAQ is available in different languages at www.ipaq.ki.se (https://sites.google.com/site/theipaq/). In this study, a short Korean version of the IPAQ of which reliability and validity was tested and reported previously [18] was used. In brief, participants were asked about frequency (times/week) and duration (minutes) of PA lasting for at least 10 minutes according to intensity (light, moderate, and vigorous). Total PA per week was estimated by summing the minutes spent in moderate PA per week and twice the minutes spent in vigorous PA per week. The participants were classified as one of the two subgroups based on the WHO recommended minimum of weekly PA: (1) physically inactive (0-149 minutes of moderate PA per week or 0-74 minutes of vigorous PA per week or less than an equivalent combination of moderate-to-vigorous PA) or (2) physically active ( $\geq 150$ minutes of moderate PA per week or $\geq 75$ minutes of vigorous PA per week or an equivalent combination of moderate-tovigorous $\mathrm{PA}$ ).

Height and weight were measured with the subjects wearing light clothing and on shoes using an automate assessment equipment (DS-102, Jenix Co., Seoul, Republic of Korea) to the nearest decimal point. WC in $\mathrm{cm}$ was measured at midpoint between the bottom of rib cage and the top of the iliac crest using nonelastic tape. BMI $\left(\mathrm{kg} / \mathrm{m}^{2}\right)$ was calculated as body weight in kilograms divided by the square of height in meters. Waist-to-height ratio (WHtR) was calculated as the WC in $\mathrm{cm}$ divided by the height in $\mathrm{cm}$. For data analyses, the subjects were then classified as either normal or obesity based on the BMI cutoff point of $\geq 25 \mathrm{~kg} / \mathrm{m}^{2}[19,20]$, and they were also classified as either not central obese or central obese based on the WHtR cutoff point of $\geq 0.5$ [21].

2.4. Measurement of CIMT. Subclinical carotid atherosclerosis was assessed using carotid artery ultrasonography equipped with a 7.5-MHz linear array imaging probe (Aloka ProSound Alpha 6, Hitachi Aloka Medical America, Wallingford, CT, USA). All measurements were performed by the same radiologist blinded to the subject's clinical status, with the subject in supine position, the head turned away from the side of interest, and the neck extended slightly. The measurement was performed in the longitudinal plan on both the right and left common carotid arteries, about $2-3 \mathrm{~cm}$ proximal to the carotid bifurcation [20]. CIMT of the far wall was evaluated manually as the distance between the lumen-intima interface and the media-adventitia interface. Measurements were obtained manually from five contiguous sites at $12-\mathrm{mm}$ intervals bilaterally, and the mean of measurements was used for statistical analysis. An abnormally increased CIMT was defined as a CIMT of the upper 25 percentile $(\geq 0.71 \mathrm{~mm})$ in the entire study population

2.5. Statistical Analyses. All statistical analyses were performed using SPSS-PC version 20 (SPSS Inc., Chicago, IL, USA). Descriptive statistics of the demographic parameters were expressed as mean and standard deviation. Continuous 
variables are reported as means \pm standard deviations, and categorical variables are presented as percentages. One-way analysis of variance followed by a post hoc test (LSD), if necessary, was used to compare measured parameters between BMI and WHtR-based subgroups stratified by weekly PA. Multivariable logistic regression was used to estimate odd ratio (OR) and 95\% confidence interval (CI) of overall and central obesity stratified by weekly PA for an abnormally increased CIMT. Lastly, a multivariate linear regression analysis was conducted to identify significant determinants of an individual variation in CIMT in the study population. Statistical significance was accepted as $\mathrm{p} \leq 0.05$.

\section{Results}

Table 1 represents the descriptive statistics of study participants according to gender. Overall, men had higher values in mean age $(\mathrm{P}<0.001)$, BMI $(\mathrm{P}<0.001)$, WHtR $(\mathrm{P}<0.001)$, percent body fat $(\mathrm{P}<0.001), \mathrm{LBM}(\mathrm{P}<0.001)$, resting systolic and diastolic $\mathrm{BPs}(\mathrm{P}<0.001$ and $\mathrm{P}<0.001$, respectively), alcohol consumption $(\mathrm{P}<0.001)$, smoking rate $(\mathrm{P}<0.001)$, but higher rates of hypertension $(\mathrm{P}<0.001)$ and diabetes $(\mathrm{P}=0.009)$ in conjunction with a higher CIMT $(\mathrm{P}<0.001)$ than women.

Table 2 represents the measured parameters of BMIbased subgroups stratified by weekly PA. Among physically active individuals, those with BMI of $\geq 25 \mathrm{~kg} / \mathrm{m}^{2}$ had higher values in WHtR $(\mathrm{P}<0.001)$, percent body fat $(\mathrm{P}<0.001), \mathrm{LBM}$ $(\mathrm{P}<0.001)$, systolic and diastolic $\mathrm{BPs}(\mathrm{P}<0.001$ and $\mathrm{P}<0.001$, respectively), smoking rate $(\mathrm{P}<0.001)$, and prevalence of hypertension $(\mathrm{P}=0.013)$ in conjunction with a higher CIMT than those with BMI of $<25 \mathrm{~kg} / \mathrm{m}^{2}$, with no statistically significance differences in mean age, alcohol consumption, and prevalence of diabetes and hypertension between the two subgroups. Among physically inactive individuals, those with BMI of $\geq 25 \mathrm{~kg} / \mathrm{m}^{2}$ had higher values in mean age $(\mathrm{P}<0.001)$, WHtR $(\mathrm{P}<0.001)$, percent body fat $(\mathrm{P}<0.001)$, LBM $(\mathrm{P}<0.001)$, systolic and diastolic BPs $(\mathrm{P}<0.001$ and $\mathrm{P}<0.001)$, smoking rate $(\mathrm{P}<0.001)$, alcohol consumption $(\mathrm{P}=0.002)$, and prevalence of hypertension $(\mathrm{P}=0.013)$ in conjunction with a higher CIMT $(\mathrm{P}<0.001)$ than BMI of $<25 \mathrm{~kg} / \mathrm{m}^{2}$, with no statistically significant difference in prevalence of diabetes and hyperlipidemia between the two subgroups.

Table 3 represents the measured parameters of WHtRbased subgroups stratified by weekly PA. Among physically active individuals, those with WHtR of $\geq 0.5$ had higher values in mean age $(\mathrm{P}<0.001)$, BMI $(\mathrm{P}<0.001)$, percent body fat $(\mathrm{P}<0.001)$, LBM $(\mathrm{P}<0.001)$, systolic and diastolic BPs $(\mathrm{P}<0.001$ and $\mathrm{P}=0.001$, respectively), and smoking rate $(\mathrm{P}=0.012)$ in conjunction with a higher CIMT $(\mathrm{P}=0.001)$ than those with WHtR of $<0.5$, with no statistically significant differences in alcohol consumption and prevalence of hypertension, diabetes, and hyperlipidemia between the two subgroups. Among physically inactive individuals, those with WHtR of $\geq 0.5$ had higher values in mean age $(\mathrm{P}<0.001)$, BMI $(\mathrm{P}<0.001)$, percent body fat $(\mathrm{P}<0.001)$, systolic and diastolic $\mathrm{BPs}(\mathrm{P}<0.001$ and $\mathrm{P}<0.001$, respectively), smoking rate $(\mathrm{P}<0.001)$, alcohol consumption $(\mathrm{P}=0.022)$, and prevalence of hypertension $(\mathrm{P}<0.001)$ and hyperlipidemia $(\mathrm{P}<0.001)$ in conjunction with a higher CIMT $(\mathrm{P}<0.001)$ than those with WHtR of $<0.5$.

Table 4 represents the estimated risk of BMI-based obesity for subclinical atherosclerosis stratified by weekly PA. Among physically active individuals, there was no significant difference in the risk of subclinical atherosclerosis between BMI-based normal weight and obese groups. Among physically inactive individuals, however, BMI-based obese group had a significantly higher risk $(\mathrm{OR}=2.15,95 \% \mathrm{CI}=1.65-3.80$, $\mathrm{P}<0.001)$ of subclinical atherosclerosis, as compared to BMIbased normal weight group. The increased risk of the obese group for subclinical atherosclerosis remained significant $(\mathrm{OR}=2.50,95 \% \mathrm{CI}=1.60-3.91, \mathrm{p}<0.001)$ even after adjustments for age, WHtR, smoking, alcohol consumption, and prevalence of hypertension, diabetes, and hyperlipidemia.

Table 5 represents the estimated risk of WHtR-based central obesity for subclinical atherosclerosis stratified by weekly PA. Among physically active individuals, there was no significant difference in the risk of subclinical atherosclerosis between WHtR-based subgroups. Among physically inactive individuals; however, WHtR-based central obese group had a significantly higher risk of subclinical atherosclerosis $(\mathrm{OR}=2.31,95 \% \mathrm{CI}=1.46-3.66, \mathrm{P}<0.001)$, as compared to WHtR-based no central obese group. The increased risk of the central obese group for subclinical atherosclerosis remained significant $(\mathrm{OR}=2.08,95 \% \mathrm{CI}=1.29-3.40, \mathrm{P}=0.003)$ even after adjustments for age, BMI, smoking, alcohol consumption, hypertension, diabetes, and hyperlipidemia.

Table 6 represents the outcomes of a multivariate linear regression model for predicting CIMT. As a result, age $(\mathrm{P}<0.001)$, sex $(\mathrm{P}=0.002)$, hypertension $(\mathrm{P}=0.014)$, smoking $(\mathrm{P}=0.054)$, BMI $(\mathrm{P}<0.001)$, and physical activity $(\mathrm{P}=0.011)$ were found to be significant determinants of CIMT in the study population.

\section{Discussion}

In this cross-sectional study, we investigated the relationships of overall and central obesity with an abnormally elevated CIMT stratified by weekly PA in Korean office workers. In general, obese individuals, whether overall or central obese, had higher CVD risk factors, including higher mean age, higher body fatness, higher smoking and alcohol consumption, and higher resting blood pressures, than their nonobese counterparts. In particular, we found that the significantly higher risk of being overall or central obese for an abnormally elevated CIMT as a surrogate of subclinical atherosclerosis was found among physically inactive individuals only but not among physically active individuals, suggesting that the association between obesity and subclinical atherosclerosis is mediated by a lower PA, at least in this study population. Multivariate linear regression analyses showed that age, sex, smoking, hypertension, BMI, and physical activity were important determinants for predicting abnormally increased CIMT.

The current findings of the study support and extend those of previous studies reporting the risk of obesity for increased CIMT. CIMT correlates with BMI and WC/WHtR in children and adolescents [22], in middle-aged adults [23], 


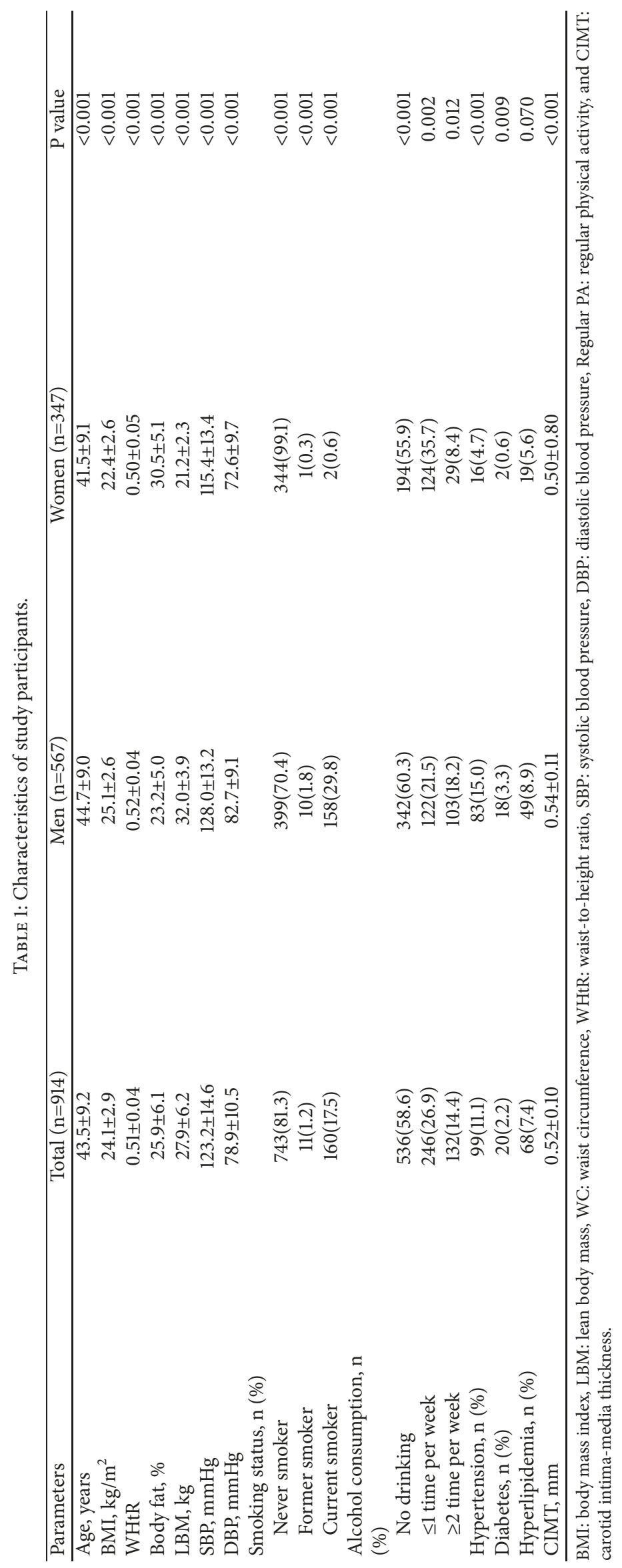




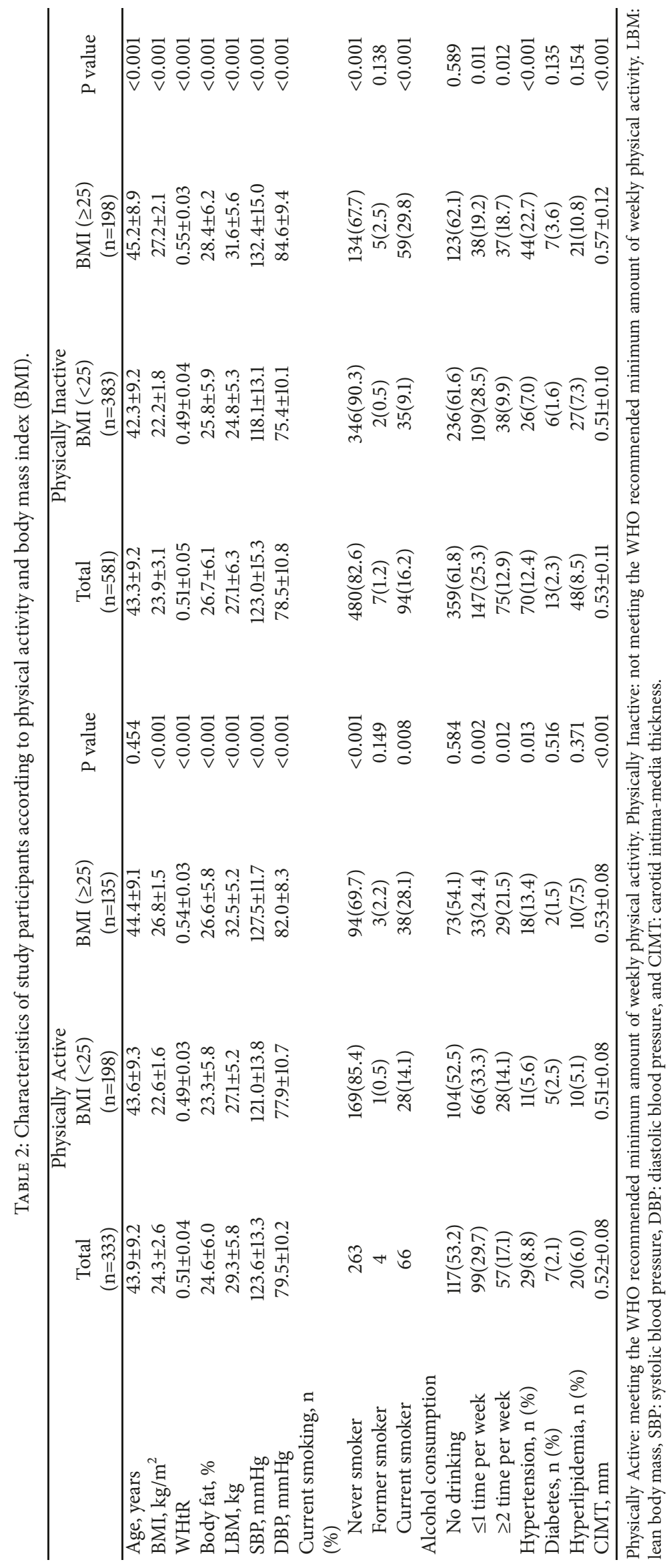




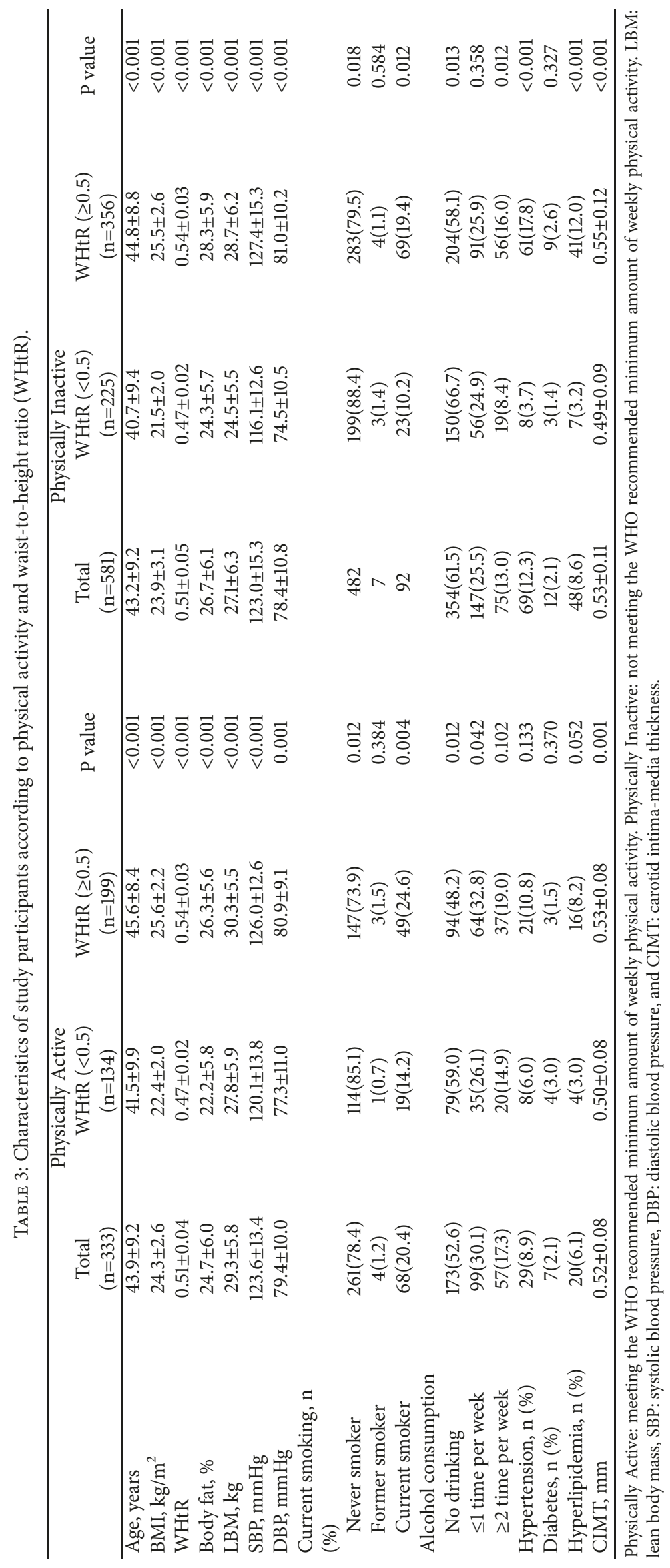


TABLE 4: Odds ratio (OR) and 95\% confidence interval (CI) according to body mass index (BMI).

\begin{tabular}{|c|c|c|c|c|c|c|c|}
\hline & & Model 1 & $\mathrm{P}$ value & Model 2 & $\mathrm{P}$ value & Model 3 & $P$ value \\
\hline \multicolumn{8}{|c|}{ Physically Active } \\
\hline \multirow{2}{*}{ BMI } & $<25 \mathrm{~kg} / \mathrm{m}^{2}$ & 1 (reference) & & 1 (reference) & & 1 (reference) & \\
\hline & $\geq 25 \mathrm{~kg} / \mathrm{m}^{2}$ & $1.38(0.81-2.35)$ & 0.240 & $1.37(0.80-2.34)$ & 0.260 & $1.36(0.78-2.36)$ & 0.274 \\
\hline \multicolumn{8}{|c|}{ Physically Inactive } \\
\hline \multirow{2}{*}{ BMI } & $<25 \mathrm{~kg} / \mathrm{m}^{2}$ & 1 (reference) & & 1 (reference) & & 1 (reference) & \\
\hline & $\geq 25 \mathrm{~kg} / \mathrm{m}^{2}$ & $2.51(1.65-3.80)$ & $<0.001$ & $2.65(1.71-4.10)$ & $<0.001$ & $2.50(1.60-3.91)$ & $<0.001$ \\
\hline
\end{tabular}

Physically Active: meeting the WHO recommended minimum amount of weekly physical activity. Physically Inactive: not meeting the WHO recommended minimum amount of weekly physical activity.

Model 1 adjusted for age and sex.

Model 2 adjusted for Model 1 plus WHtR, smoking, and alcohol consumption.

Model 3 adjusted for Model 2 plus prevalence of hypertension, diabetes, and hyperlipidemia.

TABLE 5: Odds ratio (OR) and 95\% confidence interval (CI) according to waist-to-height ratio (WHtR).

\begin{tabular}{|c|c|c|c|c|c|c|c|}
\hline & & Model 1 & $P$ value & Model 2 & $P$ value & Model 3 & $P$ value \\
\hline \multicolumn{8}{|c|}{ Physically Active } \\
\hline \multirow{2}{*}{ WHtR } & $\leq 0.50$ & 1 (reference) & & 1 (reference) & & 1 (reference) & 0.343 \\
\hline & $>0.50$ & $1.32(0.75-2.33)$ & 0.338 & $1.30(0.73-2.31)$ & 0.370 & $1.33(0.74-2.37)$ & \\
\hline \multicolumn{8}{|c|}{ Physically Inactive } \\
\hline \multirow{2}{*}{ WHtR } & $\leq 0.50$ & 1 (reference) & & 1 (reference) & & 1 (reference) & \\
\hline & $>0.50$ & $2.31(1.46-3.66)$ & $<0.001$ & $2.26(1.40-3.65)$ & 0.001 & $2.08(1.29-3.40)$ & 0.003 \\
\hline
\end{tabular}

Physically Active: meeting the WHO recommended minimum amount of weekly physical activity. Physically Inactive: not meeting the WHO recommended minimum amount of weekly physical activity.

Model 1 adjusted for age and sex.

Model 2 adjusted for Model 1 plus BMI, smoking, and alcohol consumption.

Model 3 adjusted for Model 2 plus prevalence of hypertension, diabetes, and hyperlipidemia.

TABLE 6: Multivariate linear regression for predicting carotid-intima media thickness.

\begin{tabular}{lccc}
\hline Variables & $\beta$ & $95 \%$ CI & P value \\
\hline Age & 0.399 & $0.004 \sim 0.005$ & $<0.001$ \\
Sex & -0.045 & $-0.024 \sim 0.005$ & 0.002 \\
BMI & 0.233 & $0.004 \sim 0.012$ & $<0.001$ \\
WHtR & -0.046 & $-0.340 \sim 0.130$ & 0.380 \\
Hypertension & 0.084 & $0.008 \sim 0.047$ & 0.014 \\
Smoking & 0.101 & $-0.043 \sim-0.011$ & 0.054 \\
Physical activity & -0.086 & $-0.014 \sim-0.002$ & 0.011 \\
\hline
\end{tabular}

BMI: body mass index, WHtR: waist-to-height ratio, and smoking: past and current smokers.

Regression model F-value $=54.438$, adjusted $\mathrm{R}^{2}=0.274$, and $\mathrm{P}$-value $=0.001$.

and in older adults [24]. In a sample of Korean older women, Park et al. [25] also showed overweight and obese women had significantly higher risks of subclinical atherosclerosis $(\mathrm{OR}=3.033,95 \% \mathrm{CI}=1.064-8.650$ and $\mathrm{OR}=2.459,95 \% \mathrm{CI}=$ 0.998-6.059, respectively) after adjustments for age, height, systolic blood pressure, physical activity level, and total cholesterol level, as compared with optimal weight women. In addition, central obesity was found to be more closely associated with an abnormally increased CIMT in middleaged and elderly Chinese [24]. In the current study, likewise, we also found the independent and additive roles of BMI and WHtR in determining the risk of subclinical atherosclerosis in Korean office workers. Together, those findings suggest that, in addition to total body fat, body fat distribution should be considered in determining the risk of obesity for an abnormally increased CIMT.

With respect to PA and its relation to the atherosclerosis risk, Umbreen et al. [26] showed that leisure-time physical activity was significantly associated with CIMT in a crosssectional study of 110 Pakistani adults (mean age of $58.6 \pm$ 6.7 years). Galetta et al. [27] investigated the effect of physical activity on heart rate variability (HRV) and carotid intimamedia thickness (IMT) by comparing 32 elderly sedentary subjects and 32 age-matched endurance athletes. In that study, they found that the elderly athletes had significantly lower CIMT than their sedentary counterparts. Regular PA was also found to delay the progression of CIMT in patients with coronary heart disease [28], in patients with stage 1 
hypertension [29], and in patients with type 2 diabetes [30]. In support of the findings from previous cross-sectional and longitudinal studies, intervention studies have provided positive results on the ability of physical activity including regular exercise to reverse subclinical atherosclerosis in children [31,32] as well as in older adults [33, 34]. For example, Woo et al. [32] investigated the effects of diet and/or exercise intervention programs in a sample of overweight children aged 9-12 years, and they found that obesity-related vascular dysfunction was partially reversed with the diet program alone and even more substantially with diet plus exercise program. Likewise, Park, Kwon, and Park [34] showed that 24 weeks of aerobic and resistance training resulted in significantly decreased CIMT and increased carotid flow velocity wall shear ration in a sample of overweight and obese older women. Together, these findings suggest that PA, including regular exercise, plays an important role in delaying and/or preventing the progression of atherosclerotic lesions in young and older subjects as well as in patients with CVD and metabolic disorders.

In particular, based on our knowledge on review of literatures, we are the first to report that the risk of obesity for an abnormally increased CIMT is significantly mediated by increased regular weekly PA in this study population of Korean office workers. Several explanations can be given for the mediating effect of weekly PA on the association between obesity and subclinical atherosclerosis. First, regular PA induces the beneficial effects on overweight/obesity, blood lipids, insulin resistance and diabetes, and hypertension [35], contributing to vascular health and reduced CVD incidence. Second, high cardiorespiratory fitness (CRF) provides a protection from being exposing to CVD risk and atherosclerotic lesions. Recent PA is associated with and may reflect individuals' CRF levels [36]. Consequently, the mediating effect of increased regular PA may reflect the benefits of promotion of CRF, CVD risk factors, and subclinical atherosclerosis [37]. On the other hand, lower levels of CRF are associated with morphologic and biochemical conditions that may reflect a primary atherosclerotic development [37]. Third, adipocytes produce a number of inflammatory cytokines, which may lead to systemic inflammation [38]. In this aspect, CIMT may represent an inflammatory process preceding the atheromatous plaque formation and atherosclerosis [39], which can be explained by the combination of negative profile of obesity and its related metabolic complications. On the other hand, regular PA may increase anti-inflammatory cytokines such as adiponectin and improve endothelial function [40]. Lastly, the mediating effect of regular PA on the association between obesity and increased CIMT may be a consequence of the nonatherosclerotic enlargement of carotid artery as an adaptive response to PA [41], through a nitric oxide-arteriogenesis-related mechanism [42]. Together, we speculate that the beneficial effects of regular PA on obesity, insulin resistance and metabolic complications, CVD risk factors, and inflammatory cytokines would collectively contribute to the alleviated risk of obesity for an abnormally increased CIMT as an early indicator of atherosclerosis.
This study has some limitations. Firstly, several major CVD risk factors such as blood lipids, insulin, and proinflammatory cytokines may play as additional modulators in determining the association between PA, obesity, and increased CIMT, and they should be accounted for in a future study. Secondly, the participants of the current study were all white collar office workers from Seoul, the capital city of Korea, who may not properly represent the overall office workers in Korea. Therefore, the current findings of the study may have a limitation in generalizing to Korean office workers. Thirdly, the cross-sectional nature of the current study did not allow us to suggest the exact mechanism(s) underlying those benefits, especially with respect to subclinical atherosclerosis, and this should be investigated in a future study. Lastly, the method of measuring CIMT manually used in this study may be subjected to bias and suboptimal reproducibility.

\section{Conclusion}

In this study, we investigated the association between overall and central obesity and CIMT stratified by week PA levels in Korean office workers and found that either overall or central obesity is significantly associated with increased CIMT among physical inactive individuals but not among physically active individuals. Finally, the current findings of the study suggest that, along with demographics (i.e., age and male sex) and hypertension, smoking, obesity, and physical inactivity may collectively contribute to subclinical atherosclerosis, implying an importance of promoting a healthy lifestyle for the prevention of atherosclerosis in this study population.

\section{Data Availability}

The data used to support the findings of this study are included within the article.

\section{Conflicts of Interest}

The authors declare that they have no conflicts of interest.

\section{Acknowledgments}

The National Research Foundation Grant funded by the Korean Government (NRF-2018R1D1A1B07048210) supported this work.

\section{References}

[1] Ministry of Health \& Welfare, "The physical activity guide for Koreans 2013," Sejong, Ministry of Health \& Welfare, http://www.mohw.go.kr/eng/, [accessed 25 January 2018].

[2] T. Ahn, "Reduction of Working Time: Does It Lead to a Healthy Lifestyle?" Health Economics, vol. 25, no. 8, pp. 969-983, 2016.

[3] T. W. Taris, J. F. Ybema, D. G. J. Beckers, M. W. Verheijden, S. A. E. Geurts, and M. A. J. Kompier, "Investigating the associations among overtime work, health behaviors, and health: A longitudinal study among full-time employees," International Journal of Behavioral Medicine, vol. 18, no. 4, pp. 352-360, 2011. 
[4] S. A. Peters and M. L. Bots, "Carotid intima-media thickness studies: study design and data analysis," Journal of Stroke, vol. 15, no. 1, pp. 38-48, 2013.

[5] C. Lamotte, C. Iliescu, C. Libersa, and F. Gottrand, "Increased intima-media thickness of the carotid artery in childhood: A systematic review of observational studies," European Journal of Pediatrics, vol. 170, no. 6, pp. 719-729, 2011.

[6] L. E. Chambless, G. Heiss, A. R. Folsom et al., "Association of coronary heart disease incidence with carotid arterial wall thickness and major risk factors: the Atherosclerosis Risk in Communities (ARIC) study, 1987-1993," American Journal of Epidemiology, vol. 146, no. 6, pp. 483-494, 1997.

[7] D. H. O’Leary, J. F. Polak, R. A. Kronmal et al., "Carotid-artery intima and media thickness as a risk factor for myocardial infarction and stroke in older adults," The New England Journal of Medicine, vol. 340, no. 1, pp. 14-22, 1999.

[8] S. D. Reilly, S. H. Litovsky, M. P. Steinkampf, and J. B. Caulfield, "Statins improve human coronary atherosclerotic plaque morphology," Texas Heart Institute Journal, vol. 35, no. 2, pp. 99-103, 2008.

[9] A. J. Taylor, S. M. Kent, P. J. Flaherty, L. C. Coyle, T. T. Markwood, and M. N. Vernalis, "ARBITER: Arterial biology for the investigation of the treatment effects of reducing cholesterol: A randomized trial comparing the effects of atorvastatin and pravastatin on carotid intima medial thickness," Circulation, vol. 106, no. 16, pp. 2055-2060, 2002.

[10] World Health Organization (WHO), Global Health Risks: Mortality And Burden of Disease Attributable to Selected Major Risks, WHO Library Cataloguing-in-Publication Data, 2009.

[11] R. H. Eckel and R. M. Krauss, "American Heart Association call to action: obesity as a major risk factor for coronary heart disease," AHA Nutrition Committee Circulation, vol. 97, no. 21, pp. 2099-2100, 1998.

[12] S. Kodama, C. Horikawa, K. Fujihara et al., "Comparisons of the strength of associations with future type 2 diabetes risk among anthropometric obesity indicators, including waist-to-height ratio: A meta-analysis," American Journal of Epidemiology, vol. 176, no. 11, pp. 959-969, 2012.

[13] T. Y. Warren, V. Barry, S. P. Hooker, X. Sui, T. S. Church, and S. N. Blair, "Sedentary behaviors increase risk of cardiovascular disease mortality in men," Medicine \& Science in Sports \& Exercise, vol. 42, no. 5, pp. 879-885, 2010.

[14] D. E. R. Warburton, C. W. Nicol, and S. S. D. Bredin, "Health benefits of physical activity: the evidence," Canadian Medical Association Journal, vol. 174, no. 6, pp. 801-809, 2006.

[15] G.-S. Kim, E. Im, and J.-H. Rhee, "Association of physical activity on body composition, cardiometabolic risk factors, and prevalence of cardiovascular disease in the Korean population (from the fifth Korea national health and nutrition examination survey, 2008-2011)," BMC Public Health, vol. 17, no. 1, p. 275, 2017.

[16] H. Ryu, J. Jung, J. Cho, and D. L. Chin, "Program development and effectiveness of workplace health promotion program for preventing metabolic syndrome among office workers," International Journal of Environmental Research and Public Health, vol. 14, no. 8, 2017.

[17] P. C. Hallal and C. G. Victora, "Reliability and validity of the international physical activity questionnaire (IPAQ)," Medicine \& Science in Sports \& Exercise, vol. 36, no. 3, p. 556, 2004.

[18] J. Y. Oh, Y. J. Yang, B. S. Kim, and J. H. Kang, "Validity and reliability of korean version of international physical activity questionnaire (IPAQ) short form," Korean Journal of Family Medicine, vol. 28, no. 27, pp. 532-541, 2007.
[19] P. Gordon-Larsen, L. S. Adair, J. B. Meigs et al., "Discordant risk: Overweight and cardiometabolic risk in Chinese adults," Obesity (Silver Spring), vol. 21, no. 1, pp. E166-E174, 2013.

[20] WHO Expert Consultation, "Appropriate body-mass index for asian populations and its implications for policy and intervention," Lancet, vol. 363, no. 9403, pp. 157-163, 2004.

[21] M. Ashwell and S. Gibson, "Waist to height ratio is a simple and effective obesity screening tool for cardiovascular risk factors: Analysis of data from the british national diet and nutrition survey of adults aged 19-64 years," Obesity Facts, vol. 2, no. 2, pp. 97-103, 2009.

[22] O. Elkiran, E. Yilmaz, M. Koc, A. Kamanli, B. Ustundag, and N. Ilhan, "The association between intima media thickness, central obesity and diastolic blood pressure in obese and owerweight children: A cross-sectional school-based study," International Journal of Cardiology, vol. 165, no. 3, pp. 528-532, 2013.

[23] W. Ge, F. Parvez, F. Wu et al., "Association between anthropometric measures of obesity and subclinical atherosclerosis in Bangladesh," Atherosclerosis, vol. 232, no. 1, pp. 234-241, 2014.

[24] C. Ren, J. Zhang, Y. Xu et al., "Association between carotid intima-media thickness and index of central fat distribution in middle-aged and elderly Chinese," Cardiovascular Diabetology, vol. 13, no. 1, p. 139, 2014.

[25] J.-K. Park, H. Park, and K.-B. Kim, “The relationship between distribution of body fat mass and carotid artery intima-media thickness in Korean older adults," Journal of Physical Therapy Science, vol. 27, no. 10, pp. 3141-3146, 2015.

[26] S. Umbreen, N. Khan, and G. Khan, "Association between carotid intima-media thickness and physical activity scores in middle aged Pakistani adults-a hospital based study," Journal of Ayub Medical College Abbottabad, vol. 26, no. 1, pp. 26-28, 2014.

[27] F. Galetta, F. Franzoni, L. Tocchini et al., "Effect of physical activity on heart rate variability and carotid intima-media thickness in older people," Internal and Emergency Medicine, vol. 8, Suppl 1, no. 1, pp. S27-S29, 2013.

[28] S. Sato, S. Makita, R. Uchida, S. Ishihara, and M. Majima, "Physical activity and progression of carotid intima-media thickness in patients with coronary heart disease," Journal of Cardiology, vol. 51, no. 3, pp. 157-162, 2008

[29] P. Palatini, M. Puato, M. Rattazzi, and P. Pauletto, "Effect of regular physical activity on carotid intima-media thickness. Results from a 6-year prospective study in the early stage of hypertension," Blood Pressure, vol. 20, no. 1, pp. 37-44, 2011.

[30] T. Watarai, Y. Yamasaki, M. Ikeda et al., "Insulin resistance contributes to carotid arterial wall thickness in patients with non-insulin-dependent-diabetes mellitus," Endocrine Journal, vol. 46, no. 5, pp. 629-638, 1999.

[31] A. A. Meyer, G. Kundt, U. Lenschow, P. Schuff-Werner, and W. Kienast, "Improvement of early vascular changes and cardiovascular risk factors in obese children after a six-month exercise program," Journal of the American College of Cardiology, vol. 48, no. 9, pp. 1865-1870, 2006.

[32] K. S. Woo, P. Chook, C. W. Yu et al., "Effects of Diet and Exercise on Obesity-Related Vascular Dysfunction in Children," Circulation, vol. 109, no. 16, pp. 1981-1986, 2004.

[33] J. Park and H. Park, "Effects of 6 months of aerobic and resistance exercise training on carotid artery intima media thickness in overweight and obese older women," Geriatrics \& Gerontology International, vol. 17, no. 12, pp. 2304-2310, 2017.

[34] J. Park, Y. Kwon, and H. Park, "Effects of 24-week aerobic and resistance training on carotid artery intima-media thickness 
and flow velocity in elderly women with sarcopenic obesity," Journal of Atherosclerosis and Thrombosis, vol. 24, no. 11, pp. 1117-1124, 2017.

[35] B. Strasser, "Physical activity in obesity and metabolic syndrome," Annals of the New York Academy of Sciences, vol. 1281, no. 1, pp. 141-159, 2013.

[36] A. Prioreschi, S. Brage, K. Westgate, S. A. Norris, and L. K. Micklesfield, "Cardiorespiratory fitness levels and associations with physical activity and body composition in young South African adults from Soweto," BMC Public Health, vol. 17, no. 1, p. 301, 2017.

[37] D. P. Bailey, L. M. Boddy, L. A. Savory, S. J. Denton, and C. J. Kerr, "Associations between cardiorespiratory fitness, physical activity and clustered cardiometabolic risk in children and adolescents: the HAPPY study," European Journal of Pediatrics, vol. 171, no. 9, pp. 1317-1323, 2012.

[38] Z. Wang and T. Nakayama, "Inflammation, a link between obesity and cardiovascular disease," Mediators Inflamm, vol. 2010, Article ID 535918, 2010.

[39] A. V. Finn, F. D. Kolodgie, and R. Virmani, "Correlation between carotid intimal/medial thickness and atherosclerosis: A point of view from pathology," Arteriosclerosis, Thrombosis, and Vascular Biology, vol. 30, no. 2, pp. 177-181, 2010.

[40] E. T. de Lemos, J. Oliveira, J. P. Pinheiro, and F. Reis, "Regular physical exercise as a strategy to improve antioxidant and anti-inflammatory status: benefits in type 2 diabetes mellitus," Oxidative Medicine and Cellular Longevity, vol. 2012, Article ID 741545, 2012.

[41] P.-J. Touboul, M. G. Hennerici, S. Meairs et al., "Mannheim carotid intima-media thickness consensus (2004-2006): an update on behalf of the advisory board of the 3rd and 4th Watching the Risk Symposium, 13th and 15th European Stroke Conferences, Mannheim, Germany, 2004, and Brussels, Belgium, 2006," Cerebrovascular Disease, vol. 23, no. 1, pp. 75-80, 2007.

[42] B. M. Prior, H. T. Yang, and R. L. Terjung, "What makes vessels grow with exercise training?" Journal of Applied Physiology, vol. 97, no. 3, pp. 1119-1128, 2004. 


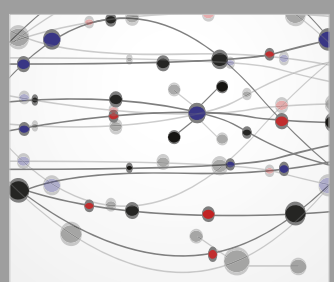

The Scientific World Journal
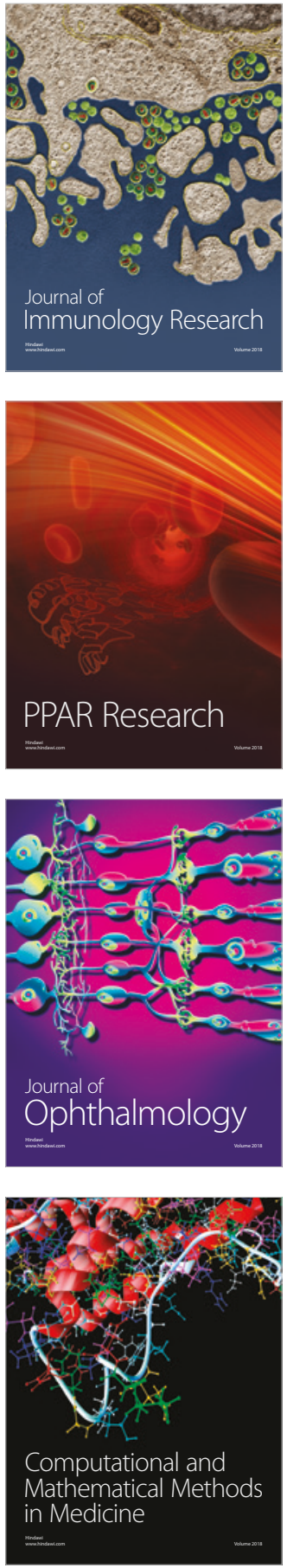

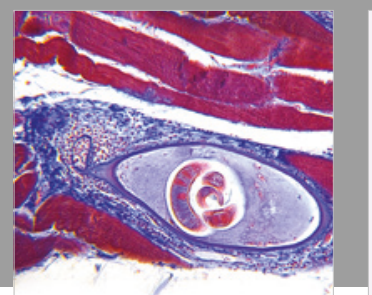

Gastroenterology Research and Practice

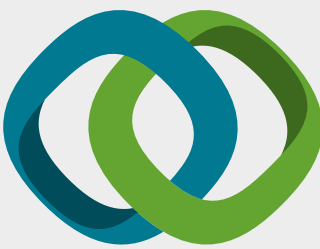

\section{Hindawi}

Submit your manuscripts at

www.hindawi.com
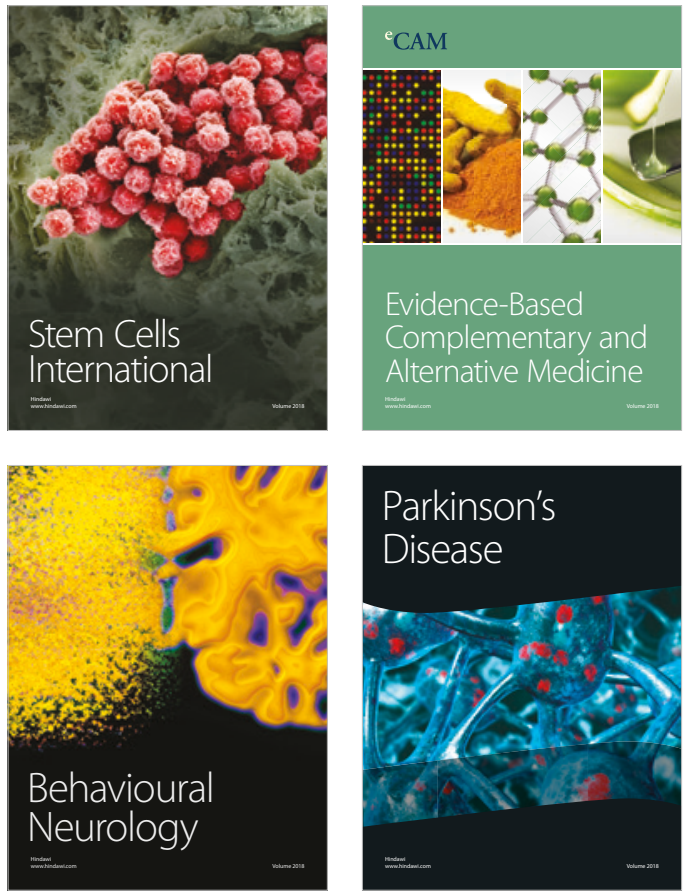

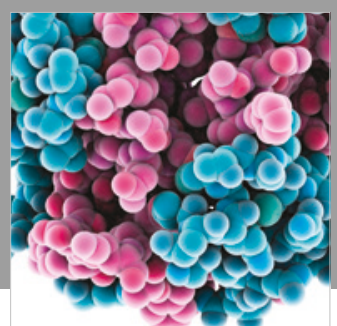

ournal of

Diabetes Research

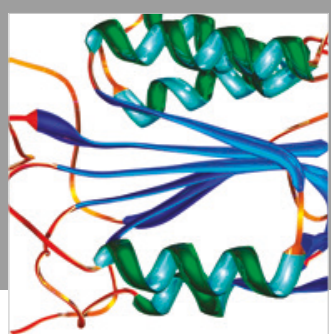

Disease Markers
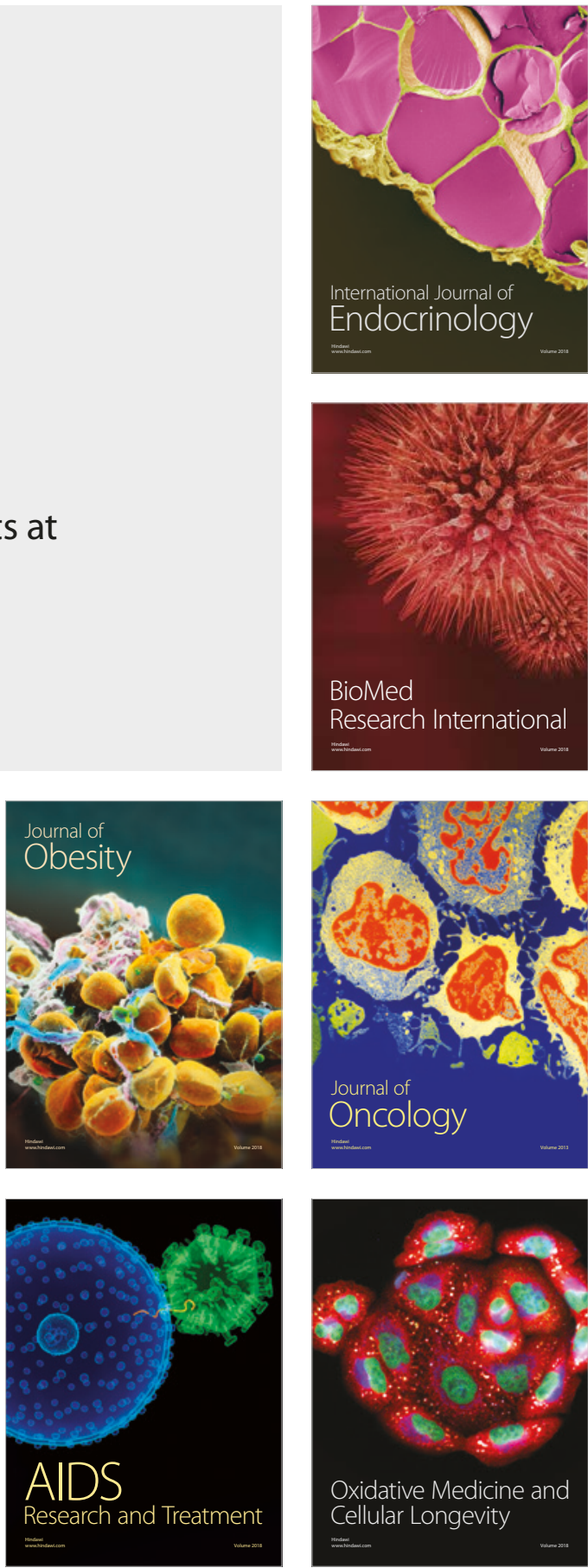